\title{
DIFFERENTIAL FORMS, WEITZENBÖCK FORMULAE AND FOLIATIONS
}

\author{
HANSKLAUS RUMMLER.
}

\begin{abstract}
The Weitzenböcl formulae express the Laplacian of a differential form on an oriented Riemannian manifold in local coordinates, using the covariant derivatives of the form and the coefficients of the curvature tensor. In the first part, we shall describe a certain "differential algebra formalism" which seems to be a more natural frame for those formulae than the usual calculations in local coordinates.

In this formalism, there appear some interesting differential operators which may also be used to characterize local geometric properties of foliations. That is the topic of the second part.
\end{abstract}

\section{Differential Forms and Weitzenböck Formulae}

\section{Introduction.}

Relations between the curvature tensor of a compact oriented Riemannian manifold and its topology play an important role in global differential geometry. The oldest and still most beautiful theorem of this kind is of course the global Gauß-Bonnet formula. Another theorem of this type is the following one by $S$. Bochner (see [3],[1]): If all sectional curvatures are positive, then the first. Betti number must vanish. To prove this theorem, one can for instance show that there are no non-trivial harmonic 1 -forms, and in order to derive this from the positivity of sectional curvature, one uses the so-called Weitzenböck formulae, expressing the Laplacian of a differential form in local coordinates by an expression which contains coefficients of the curvature tensor. In order to see, how the curvature tensor comes in, we shall develop an appropriate formalism in the next two paragraphs.

The author would like to thank the organizers of the Differential Geometry Semester in autumn 1988 at the Centre de Recerca Matematica of the Institut d'Estudis Catalans, where this article was written. 
2. Ordinary and Vector-valued Differential Forms.

Let $M$ be a differentiable (i.e. $C^{\infty}$ ) manifold, $T M$ its tangent bundle and $T^{*} M$ the cotangent bundle. A vector-yalued $p$-form $\Phi$ on $M$ is a differentiable (i.e. $C^{\infty}$ ) section in the bundle $T M \otimes \wedge^{p} T^{*} M$. Locally, $\Phi$ may be written as

$$
\Phi=\sum_{i=1}^{n} \varphi_{i} \otimes X_{i}
$$

with ordinary $p$-forms $\varphi_{i}$ and vector fields $X_{i}$. For $x \in M$ one can interpret $\Phi(x)$ as a $p$-linear alternating map from $T_{x} M \times \ldots \times T_{x} M$ to $T_{x} M$.

The vector space of these forms will be denoted by $\vec{A}^{p}=\vec{A}^{p}(M)$, and we shall write $A^{p}=A^{p}(M)$ for that of ordinary p-forms. $\vec{A}=\bigoplus_{p=0}^{n} \vec{A}^{p}$ is in a natural way an $A$-module, i.e. there are exterior products

$$
\wedge: A^{p} \times \vec{A}^{q} \longrightarrow \overrightarrow{A^{p+q}} \quad \text { and } \quad \wedge: \vec{A}^{q} \times A^{p} \longrightarrow \vec{A}^{p+q},
$$

with the obvious commutation rules.

Now let $M$ be a Riemannian manifold. Then the Riemannian metric induces also an "exterior inner product"

$$
\langle,\rangle: \vec{A}^{p} \times \vec{A}^{q} \rightarrow A^{p+q},
$$

and there is also a Hodge *-operator

$$
\star: \vec{A}^{p} \longrightarrow \vec{A}^{n-p},
$$

locally defined by $\star\left(\sum \varphi_{i} \otimes X_{i}\right)=\sum\left(\star \varphi_{i}\right) \otimes X_{i}$.

Let $D$ denote the Levi-Civita connection on $M$. It can be interpreted as a map $D: \vec{A}^{0} \longrightarrow \vec{A}^{3}$, and this map is extended in a unique way by maps $D: \vec{A}^{p} \longrightarrow \vec{A}^{p+1}$, such that the product rule

$$
D(\varphi \wedge \Psi)=d \varphi \wedge \Psi+(-1)^{p} \varphi \wedge D \Psi
$$

holds for $\varphi \in A^{p}$ and $\Psi \in \vec{A}^{\varphi}$.

This can be done for any linear connection on $T M$, but the Levi-Civita connection satisfies also the product rule for our "exterior inner product":

$$
d\langle\Phi, \Psi\rangle=\langle D \Phi, \Psi\rangle+(-1)^{p}\langle\Phi, D \Psi\rangle
$$

for $\Phi \in \vec{A}^{p}$ and $\Psi \in \vec{A}^{q}$.

In this formalism, the curvature tensor is simply given by

$$
R_{X Y} Z=D^{2} Z(X, Y) \quad \text { for } \quad X, Y, Z \in \vec{A}^{0},
$$


and also the torsion (which vanishes in one case) has a simple interpretation: $T_{D}=D i d_{T M}$, where the identity on the tangent bundle $T M$ is interpreted as a vector-valued 1-form.

The Riemanmian metric induces also several maps between ordinary and vector-valued differential forms, which we shall now describe: The first one consist of maps

$$
j: A^{p} \longrightarrow \vec{A}^{p-1}
$$

which extend the canonical isomorphism between 1 -forms and vector fields. $j$ is simply defined by $\langle j \omega, X\rangle=i_{X} \omega$ for $\omega \in A^{p}$ and $X \in \vec{A}^{0}$, where $i_{X}$ denotes the interior derivative. $j$ is a derivation of degree -1 , i.e. it satisfies the product rule

$$
j(\varphi \wedge \psi)=j \varphi \wedge \psi+(-1)^{p} \varphi \wedge j \psi
$$

for $\varphi \in A^{p}$ and $\psi \in A^{4}$. We can as well extend the inverse isomorphism $\alpha=j^{-1}: \vec{A}^{0} \longrightarrow A^{1}$ by

$$
\alpha \Phi\left(X_{1}, \ldots, X_{p+1}\right)=\sum_{i=1}^{p+1}(-1)^{i+1}\left\langle X_{1}, \Phi\left(X_{1}, \ldots, \hat{X}_{i}, \ldots, X_{p+1}\right)\right\rangle .
$$

The next map is the contraction with respect to the first argument, i.e. the map "trace" tr : $\vec{A}^{p} \longrightarrow A^{p-1}$, defined by

$$
\operatorname{tr} \Phi\left(X_{1}, \ldots, X_{p-1}\right)=\text { trace }\left(\Phi\left(-, X_{1}, \ldots, X_{p-1}\right): T M \longrightarrow T M\right),
$$

and the last map is a sort of a "diagonal" diag : $A^{p} \longrightarrow \vec{A}^{p+1}$, an extension of the map from $A^{0}$ to $\vec{A}^{1}$ that sends the constant 1 to the identity $i d_{T M}$.

We shall not derive all the relations between those maps, but we mention that the following two diagrams commute up to sign:

$$
\begin{aligned}
& A^{p} \longleftarrow \quad \longrightarrow A^{n-p} \quad A^{p} \longmapsto A^{n-n} \\
& \vec{A}^{p+1} \longmapsto \vec{A}^{n-p-1} \quad \vec{A}^{p-1} \longleftarrow \quad \longrightarrow \vec{A}^{p-p+1}
\end{aligned}
$$

\section{Gradient, Divergence and Laplacian.}

In our language, the classical operators grad and div may be expressed by

$$
\text { grad }=j d: A^{0} \longrightarrow \vec{A}^{0} \text { and } \quad d i v=\operatorname{tr} D: \vec{A}^{0} \longrightarrow A^{0} .
$$

As by defnition - $j$ vanishes on $A^{0}$ and $t r$ on $\vec{A}^{0}$, we may as well use the "more symmetric" formulac.

$$
\operatorname{grad}=j d+D j \quad \text { and } \quad d i v=t r D+d t r,
$$

and we use these formulae to define gradient and divergence in any degree $p$ for $0 \leq p \leq n$.

The reason for adding the terms $D, j$ and $d t r$ which are "hidden" in degree zero is of course not only an esthetic one, but it is the following theorem: 
Theorem 1. The operators grad and div satisfy the following relations, by which they are uniquely determined:

(1) $f \in A^{0} \Longrightarrow$ grad $f$ is the ordinary gradient

(2) $f \in A^{0} \Longrightarrow \operatorname{gradd} f=D$ gradf

(3) $\varphi \in A^{p}, \psi \in A^{q} \Longrightarrow \operatorname{grad}(\varphi \wedge \psi)=(\operatorname{grad} \varphi) \wedge \psi+\varphi \wedge \operatorname{grad} \psi$

(4) $X \in \vec{A}^{0} \Longrightarrow$ div $X$ is the ordinary divergence

(5) $\varphi \in A^{p}, \psi \in \vec{A}^{g} \Longrightarrow \operatorname{div}(\varphi \wedge \Psi)=\langle\operatorname{grad} \varphi, \Psi\rangle+\varphi \wedge \operatorname{div} \Psi$

We shall not prove this theorem - nor the next one - but only indicate that this can be done using local coordinates which are geodesic at a given point $x \in M$. Observe also that property (2) is not valid in degree $p>0$, where we have $D$ grad $\omega-\operatorname{grad} d \omega=D^{2} j \omega$, which does not vanish in general if the curvature tensor is non-zero.

The properties (1) - (3) of the gradient and the well-known rules for covariant derivation imply:

Corollary. For $\varphi \in A^{p}$ and $X \in \vec{A}^{0}$,

$$
\langle X, \operatorname{grad} \varphi\rangle=D_{X} \varphi, \text { the covariant derivative. }
$$

This characterizes grad $\varphi$ completely.

So the gradient grad $\omega$ contains the information about all the covariant derivatives of the $p$-form $\omega$, and is thus metrically equivalent to the tensor $D \omega$, defined by $D \omega\left(X ; X_{1}, \ldots, X_{p}\right)=\left(D_{X} \omega\right)\left(X_{1}, \ldots, X_{p}\right)$. So why not simply use this tensor instead of the vector-valued form grad $\omega$ ? One good reason is that this tensor is only alternating in the last $p$ arguments, and by changing the variance of the first argument we exhibit its special role. (Antisymmetrizing $D \omega$ would give the ordinary exterior derivative $d \omega$, because the Levi-Civita connection $D$ is torsion-free.)

Theorem 2. Between the operntors grad, div and the classical operators $d, \star, \delta=(-1)^{n p+n} \star d \star$, there hold the following relations:

$$
\begin{aligned}
& d=a \operatorname{grad} \\
& \delta=t r \operatorname{grad} \\
& \star \operatorname{grad}=\operatorname{grad} \star \\
& \star d i v=d i v \star
\end{aligned}
$$

Now let us try - for the sake of curiosity - whether we have also the relation $\Delta=$ div grad as in degree zero: With the relations (6) and (7) of the above theorem and the fact that $\operatorname{tr} j=0$ we get easily

$$
\operatorname{div} \operatorname{grad}=(\operatorname{tr} D+d \operatorname{tr})(j d+D j)=\delta d+d \delta+\operatorname{tr} D^{2} j=\Delta+\operatorname{tr} D^{2} j .
$$

So we don't have $\Delta=$ div grad, but we make the following definition: 
Definition. On an oriented Riemannian manifold $M$, we set

$$
\Delta_{0}:=\text { divgrad and } \quad \text { Ric }:=t r D^{2} j .
$$

Thus we have the

\section{Weitzenböck Formula.}

$$
\Delta=\Delta_{0}-R i c
$$

Of course, this is not really interesting as long as we don't know something about at least two of these three operators! To begin with, let us consider the operator Ric:

One checks easily, that Ric commutes with $*$, and that it is $A^{0}$-linear, i.e. it is a tensorial operator: for $\omega \in A^{p}$ the value Ric $\omega(x)$ at a point $x \in M$ depends only on $\omega(x)$. The name "Ric" for this operator is justified by the following: For two vector fields $X$ and $Y$, we have for the 1 -form $\omega=\alpha X$ :

$$
(\operatorname{Ric} \omega)(Y)=\left(\operatorname{tr} D^{2} X\right)(Y)=\operatorname{trace} R_{-,}, X=\operatorname{Ric}(Y, X)=\operatorname{Ric}(X, Y),
$$

the well-known Ricci tensor on $M$ !

The operator $\Delta_{0}$ commutes also with $*$, so its kernel

$$
H_{0}:=\operatorname{ker} \Delta_{0}=\bigoplus_{p=0}^{n} H_{0}^{p}
$$

is a $*$-invariant subspace of $A=\bigoplus A^{p}$. As we shall see in the next paragraph, it is even a finite-dimensional subalgebra. For the moment, we mention the product rule

$$
\Delta_{0}(\varphi \wedge \psi)=\Delta_{0} \varphi \wedge \psi+\varphi \wedge \Delta_{0} \psi+2\langle\operatorname{grad} \varphi, \operatorname{grad} \psi\rangle .
$$

\section{Compact manifolds.}

The reason for using differential forms rather than arbitrary, non-alternating tensors is that we can integrate differential forms. So let $M$ be in this paragraph a compact connected oriented Riemannian manifold of dimension $n$.

There are global inner products on $A^{p}$ and $\vec{A}^{p}$, obtained by integration of the pointwise inner products:

$$
(\varphi, \psi):=\int_{M} \varphi \wedge \star \psi \quad \text { for } \quad \varphi, \psi \in A^{p}
$$

and

$$
(\Phi, \Psi):=\int_{M}\langle\Phi, * \Psi\rangle \quad \text { for } \Phi, \Psi \in \vec{A}^{p} .
$$

The following lemma will be the key formula for the further properties we shall derive for $\Delta_{0}$ : 
Lemma. With respect to the global inner products, grad and -div are adjoints, i.e. we have for $\varphi \in A^{p}$ and $\Psi \in \vec{A}^{p}$

$$
(\operatorname{grad} \varphi, \Psi)=-(\varphi, \operatorname{div} \Psi)
$$

Proof:

$$
\begin{aligned}
(\operatorname{grad} \varphi, \Psi)+(\varphi, \operatorname{div} \Psi) & =\int_{M}(\langle\operatorname{grad} \varphi, \star \Psi\rangle+\varphi \wedge \star \operatorname{div} \Psi) \\
& =\int_{M}(\langle\operatorname{grad} \varphi, \star \Psi\rangle+\varphi \wedge \operatorname{div} \star \Psi) \\
& =\int_{M} \operatorname{div}(\varphi \wedge \star \Psi),
\end{aligned}
$$

but $\operatorname{div}(\varphi \wedge * \Psi)=\operatorname{dtr}(\varphi \wedge * \Psi)$, because the second term of the divergence, $\operatorname{tr} D(\varphi \wedge * \Psi)$, vanishes since $\varphi \wedge * \Psi$ is of maximum degree $n$. Thus, by Stokes' theorem, the result follows.

This simple integral formula has some interesting consequences. The following one is obvious:

Proposition. $\left(\Delta_{0} \omega, \omega\right) \leq 0$ for all $\omega \in A^{p}$. $\Delta$.

(Observe that with our sign convention for $\delta$ the same holds for the Laplacian

\section{Theorem 3.}

$$
\Delta_{0} \omega=0 \Longleftrightarrow \operatorname{grad} \omega=0 \Longleftrightarrow \Delta \omega=0 \text { and Ric } \omega=0 .
$$

Proof: By definition of $\Delta_{0}$, grad $\omega=0$ implies trivially $\Delta_{0} \omega=0$.

So let $\Delta_{0} \omega=0$. Then $(\operatorname{grced} \omega, \operatorname{grad} \omega)=-\left(\Delta_{0} \omega, \omega\right)=0$, and so $\operatorname{grad} \omega=0$, which implies by theorem 2 aiso $d \omega=0$ and $\delta \omega=0$, hence $\Delta \omega=0$, which, together with the hypothesis $\Delta_{0} \omega=0$, gives also Ric $\omega=0$. The remaining part is again trivial.

An immediate consequence is the following corollary, if we remember that grad is a derivation of legree zero:

Corollary, $H_{0}=\bigoplus_{p=0}^{n} H_{0}^{p}$, the kernel of $\Delta_{0}$, is a subalgebra of $A=\bigoplus A^{p}$. Moreover $H_{0}^{p} \subset H^{p}:=$ ker $\Delta \cap A^{p}$, the space of harmonic p-forms.

In particular, the space $H_{0}^{p}$ must be finite-dimensional, as $H^{p}$ is. But there is another proof of this fact, which gives still more information about the structure of the space $H_{o}^{p}$ : 
Theorem 4, For $\varphi, \psi \in H_{0}^{p}$,

$$
\langle\varphi, \psi\rangle=\text { const. }=\frac{1}{v o l M}\langle\varphi, \psi\rangle,
$$

where $\langle$,$\rangle is the pointwise scalar product on p-forms, induced by the Rie-$ mannian metric.

Proof: $\langle\varphi, \psi\rangle=*\left(\varphi_{\wedge} * \psi\right)$, and thus

$$
d\langle\varphi, \psi\rangle= \pm \star \delta(\varphi \wedge \star \psi)= \pm \star \operatorname{tr} \operatorname{grad}(\varphi \wedge \star \psi),
$$

but this expression vanishes by the product rule and $*$-invariance of grad.

This theorem says, that for any $x \in M$ the evaluation map

$$
\operatorname{eval}_{x}: H_{0}^{p} \longrightarrow \bigwedge^{p} T_{x}^{*} M
$$

is an isometric injection, up to the constant factor $1 /$ volM. As a consequence, $H_{o}^{p}$ is of dimension $\leq\left(\begin{array}{l}n \\ p\end{array}\right)$.

If $M$ is flat, we have $D^{2}=0$, hence Ric $=0$, and thus $\Delta=\Delta_{0}$. So we get in this case $\operatorname{dim} H^{p}<\infty$ without using the ellipticity of $\Delta$. (Of course, we cannot prove the regularity in this way: if we admit harmonic forms with coeffcients in a Sobolev space, we are not sure that the $C^{\infty}$ ones are already all of them.) The same property as for flat manifolds holds still in the case where Ric is positive semi-definite:

Theorem 5. If the operator Ric: $A^{p} \longrightarrow A^{p}$ is positive semi-definite, then $H_{0}^{p}=H^{p}$.

If Ric: $A^{p} \longrightarrow A^{p}$ is even positive definite, then $H_{0}^{p}=H^{p}=0$.

Proof: In the expression

$$
(\Delta \omega, \omega)=\left(\Delta_{0} \omega, \omega\right)-(\operatorname{Ric} \omega, \omega)
$$

all three terms are $\leq 0$. So, $\Delta \omega=0$ implies $\left(\Delta_{0} \omega, \omega\right)=0$, and that implies grad $\omega=0$, hence $\omega \in H_{0}^{p}$. But $($ Ric $\omega, \omega)$ must also vanish, and thus $\omega=0$ if Ric is positive definite.

The special case $p=1$ yields of course the theorem by Bochner that we mentioned in the introduction. Even if the sectional curvatures are only positive in almost all plane directions at almost all points, the ordinary Ricci tensor is positive definite, which means exactly that our Ric: $A^{1} \longrightarrow A^{1}$ is so. 


\section{The Geometry of Foliations}

\section{The characteristic form of a foliation.}

We shall be interested in local geometric properties of foliations on Riernannian manifolds. Therefore we look at the distribution in $T M$ tangent to the leaves. So, foliation means in the following simply integrable distribution (i.e. a $C^{\infty}$-subbundle) $F \subset T M$. For the sake of simplicity everything is oriented: the manifold and the foliation.

Consider the situation at a point $x \in M$ : The $p$-dimensional linear subspace $F_{x} \subset T_{x} M$ is an element of the Grassmannian manifold of all $p$-dimensional oriented linear subspaces of $T_{x} M$. But things become simpler in our case, where $T_{x} M$ is a Euclidean space: In this case, the Grassmannian becomes in a natural way a (generating) submanifold of the linear space $\wedge^{p} T_{x} M$, and $F_{x}$ is represented by $u_{1} \wedge \ldots \wedge u_{p}$, where $\left(u_{1}, \ldots, u_{p}\right)$ is a positively oriented orthonormal basis of $F_{x}$. As analysts are more used to consider the space $\wedge^{p} T_{x}^{*} M$ of $p$-forms than that of $p$-vectors, we can still use the Euclidean structure of $T_{x} M$ to identify $\wedge^{p} T_{x} M$ with $\wedge^{p} T_{x}^{*} M$ : To $F_{x}$ corresponds now the $p$-form $x \in \Lambda^{p} T_{x}^{*} M$ with

$$
\chi\left(u_{1}, \ldots, r t_{p}\right)= \begin{cases}1 & \text { for any pos. oriented orthonormal basis of } F_{x}, \\ 0 & \text { if one of the } u_{i} \text { 's is perpendicular to } F_{x} .\end{cases}
$$

Doing this at every point $x \in M$ simultaneously, we get the characteristic form $\chi_{F}$ of the foliation or distribution $F$. In the case of a foliation, the restriction of $\chi_{F}$ to a leaf is simply the volume form for that leaf.

For calculations with the characteristic form of the distribution $F$, the following simple representation is often useful: Let $\left(X_{1}, \ldots, X_{n}\right)$ be a local positively oriented orthonormal frame of vector fields on the open subset $U \subset$ $M$, such that $X_{1}, \ldots, X_{p}$ span $F$. If $\left(\omega_{1}, \ldots, \omega_{n}\right)$ denotes the dual frame of 1 -forms, we have

$$
x_{F}=\omega_{1} \wedge \ldots \wedge \omega_{p}
$$

and as $\omega_{i}\left(X_{j}\right)=\delta_{i j}$, we get for the differentials of the $\omega_{i}$ simply

$$
\begin{aligned}
d \omega_{i}\left(X_{j}, X_{k}\right) & =-\omega_{i}\left(\left[X_{j}, X_{k}\right]\right) \\
& =\left\langle X_{i},\left[X_{k}, X_{j}\right]\right\rangle \\
& =\left\langle X_{i}, D X_{k} X_{j}\right\rangle-\left\langle X_{i}, D X_{j}, X_{k}\right\rangle \\
& =\left\langle D_{X}, X_{i}, X_{k}\right\rangle-\left\langle D_{X_{k}} X_{i}, X_{j}\right\rangle
\end{aligned}
$$

2. Geometric properties of a foliation and differential properties of its characteristic form.

To begin with, and to give also an idea of what kind of calculations one has to do, let us try to find out what it means that $\chi_{F}$ is closed: 
With the above notations that means

$$
d \chi_{F}\left(X_{i_{1}}, \ldots, X_{i_{p+1}}\right)=0 \text { for } 1 \leq i_{1} \ldots i_{p+1} \leq n
$$

It is clear that $d \chi_{F}\left(X_{i_{1}}, \ldots, X_{i_{p+1}}\right)=0$ if more than two indices are $>p$. So there remain two cases to be interpreted:

$$
d \chi F\left(X_{1}, \ldots, X_{p}, X_{j}\right)=0 \text { for } p+1 \leq j \leq n
$$

and

$$
d \chi_{F}\left(X_{1}, \ldots, \hat{X}_{i}, \ldots, X_{p}, X_{j}, X_{k}\right)=0 \text { for } 1 \leq i \leq p<j<k \leq n
$$

For the first case one finds easily

$$
d \chi_{F}\left(X_{1}, \ldots, X_{p}, X_{j}\right)=(-1)^{p} \sum_{i=1}^{p}\left\langle X_{i}, D_{X_{i}} X_{j}\right\rangle=(-1)^{p+3} p\left\langle H, X_{j}\right\rangle
$$

where $H$ is the mean curvature vector field of the foliation. Thus (1) means that all leaves are minimal submanifolds. Observe that (1) says simply that the restriction of $\chi_{F}$ to any $(p+1)$-dimensional submanifold tangent to the leaves is closed.

For the second case one gets

$$
d \chi_{F}\left(X_{1}, \ldots, \hat{X}_{i}, \ldots, X_{p}, X_{j}, X_{k}\right)=(-1)^{i}\left\langle X_{i},\left[X_{j}, X_{k}\right]\right\rangle,
$$

and (2) means simply that the orthogonal complement $F^{\perp}$ is integrable. This applies also if $F$ itself is not integrable. Together with the fact that $\star \chi_{F}=\chi_{F^{1}}$, we have thus the following theorem and the two corollaries:

Theorem 6. The characteristic form of the foliation $F$ is closed if and only if all the leaves are minimal submanifolds and the orthogonal complement $F^{\perp}$ is integrable.

Corollary 1. If the characteristic form $\chi_{F}$ of the distribution $F$ is co-closed, i.e. if $\delta_{X F}=0$, then $F$ is integrable.

However, this condition is not necessary for the integrability of $F$ : If $\operatorname{dim} F=$ 1 , we have $\delta \chi_{F}=\operatorname{div} X$, where $X$ is the normed vector field describing $F$. So $\delta \chi F=0$ means in this case that the corresponding flow is volume preserving.

Corollary 2. On a compact oriented Riemannian manifold, the characteristic form $\chi_{F}$ of the distribution $F$ is harmonic if and only if $F$ and $F^{\perp}$ are foliations by minimal submanifolds.

In order to check the properties of $\operatorname{grad} \chi_{F}$, one needs essentially only the following lemma, the proof of which is trivial: 
Lemma. With the notations introduced in the last section, locally

$$
\operatorname{grad} \chi_{F}=\sum_{i=1}^{p} \omega_{1} \wedge \ldots \wedge \omega_{i-1} \wedge \operatorname{grad} \omega_{i} \wedge \omega_{i+1} \wedge \ldots \wedge \omega_{p},
$$

with $\left\langle\operatorname{grad} \omega_{i}\left(X_{j}\right), X_{k}\right\rangle=\left\langle D_{X_{k}} X_{i}, X_{j}\right\rangle$ for $1 \leq i \leq p$ and $1 \leq j, k \leq n$.

Before using this lemma, let us make two definitions:

Definition. A distribution $F \subset T M$ is said to be geodesic if $D_{X} Y$ is tangent to $F$ whenever the vector fields $X$ and $Y$ are tangent to $F$.

The distribution is said to be parallel if $D_{X} Y$ is tangent to $F$ whenever the vector field $Y$ is tangent to $F$ and the vectorfield $X$ is perpendicular to $F$.

Let us make some remarks concerning these definitions:

(1) By definition, these notions are dual: $F$ is geodesic if and only if $F^{\perp}$ is parallel.

(2) If $F$ is geodesic, it is integrable and all the leaves are totally geodesic submanifolds. The converse is also true: A foliation with totally geodesic leaves is geodesic in the sense of the above definition. However, if a distribution $F$ is totally geodesic in the sense that any geodesic tangent to $F$ at some time remains tangent to $F$ forever, the distribution $F$ needs not be integrable, and so it is not necessarily geodesic in the sense of the above definition.

(3) If $F$ is a parallel foliation, then the Riemannian metric is bundle-like for $F$, in the sense of $B$. Reinhart, but the converse is only true if $F^{\perp}$ is integrable.

(4) The terminology is inspired by the following example in $R^{2} \backslash\{0\}$ : The foliation $F$ by straight lines passing through the origin is geodesic, and the foliation $F^{\perp}$ by concentric circles around the origin is parallel.

The following two theorems are now easy consequences of the lemma, and we leave their proof as an exercise:

Theorem 7. The distribution $F$ is geodesic $\Longleftrightarrow \operatorname{grad}_{\chi F} \perp F$, i.e. all values are perpendicular to $F$, parallel $\Longleftrightarrow \operatorname{grad} \chi F \| F$, i.e. all values are tangent to $F$.

Theorem 8. Let $F$ be a foliation. Then the given Riemannian metric on $M$ is bundle-like for $F$ if and only if

$$
i_{X \operatorname{grad}} X_{F} \perp X \text { for every } X \perp F \text {. }
$$

3. Foliations of compact Riemannian manifolds.

If the manifold $M$ is compact, then $g r a d \chi_{F}=0 \Longleftrightarrow \Delta_{0} \chi_{F}=0$, and we may apply the results of the last section of the first part to get some informations about geodesic and parallel foliations. The first result is a trivial consequence of the fact that $H_{a}^{p} \subset H^{p}$ : 
Theorem 9. If the Betti number $b_{p}(M)=0$, then there is no $p$-dimensional foliation on $M$ which is at the same time geodesic and parallel, whatever is the Riemannian metric given on $M$.

In the case $p=1$ we can also say something about the set of all geodesic and parallel flows (i.e. 1-dimensional foliations) on $M$ :

Theorem 10. Let $M$ be a compact, connected, oriented Riemannian manifold, and let $p:=\operatorname{dim} H_{o}^{1}>0$. Then there is a differentiable action $\Phi: \mathbf{R}^{p} \times M \longrightarrow M$ such that

(1) $\mathbf{R}^{p}$ acts on $M$ by isometriy ;

(2) The orbits of this action are the leaves of a geodesic and parallel $p$ dimensional foliation;

(3) The projection of $\mathbf{R}^{p}$ onto any orbit is a local isometry, where $\mathbf{R}^{p}$ carries the standard Euclidean structure;

(4) Any geodesic and parallel flow on $M$ is the restriction of this action to a 1-dimensional linear subspace of $\mathrm{R}^{p}$.

Proof: Recall that the evaluation maps eval $: H_{0}^{1} \longrightarrow T_{x}^{*} M$ are isometric injections, up to a constant factor, and that also $j: T_{x}^{*} M \longrightarrow T_{x} M$ is an isometry. Thus, by choosing an appropriate basis in $H_{o}^{1}$, we get a linear map

$$
X: \mathrm{R}^{p} \longrightarrow \vec{A}^{0}
$$

with the following properties:

(i) eval $l_{x} \circ X: \mathrm{R}^{p} \rightarrow T_{x} M$ is an isometric injection for any $x \in M$, where $\mathbf{R}^{P}$ carries the standard Euclidean structure;

(ii) The 1 -form $\omega_{u}=j^{-1}\left(X_{u}\right)$ has vanishing gradient for any $u \in \mathbf{R}^{p}$.

Now, if $\|u\|=1, \omega_{u}$ is the characteristic form for the flow defined by the vector field $X_{u}$, and this flow must be geodesic and parallel. Conversely, the characteristic form of any geodesic and parallel flow on $M$ is in $H_{0}^{1}$ and hence of the form $\omega_{u}$ for some $u \in \mathbf{R}^{p}$ with $\|u\|=1$. This will prove (4) once we have shown the other properties.

We define now the action of $\mathrm{R}^{p}$ on $M$ by these flows, i.e. by

$$
\Phi(u, x):=\exp _{x}\left(X_{u}(x)\right) .
$$

To prove that this is a well-defined action, we must show that the flows defined by the vectorfields $X_{u}$ commute. So let $u, v \in \mathbf{R}^{p}$. Then

$$
D_{X_{u}} X_{v}=D X_{v}\left(X_{u}\right)=\left(D j \omega_{v}\right)\left(X_{u}\right)=\operatorname{grad} \omega_{v}\left(X_{u}\right)-j d \omega_{v}\left(X_{u}\right)=0,
$$

because with $\operatorname{grad} \omega_{1}=0$ we have also $d \omega_{v}=0$. Thus $\left[X_{u}, X_{v}\right]=D_{X_{u}} X_{v}-$ $D_{X_{v}} X_{u}=0$. 
A similar calculation shows that the vector fields $X_{u}$ are Killing fields, i.e. that $\mathbf{R}^{p}$ acts on $M$ by isometries.

If $e_{1}, \ldots, e_{p}$ are the standard basis vectors of $\mathrm{R}^{p}$, the distribution $F$ on $M$ defined by the map $X: \mathbf{R}^{p} \longrightarrow \vec{A}^{0}$ has just the characteristic form

$$
\chi_{F}=\omega_{e_{1}} \wedge \ldots \wedge \omega_{e_{p}},
$$

which has vanishing gradient. So $F$ is a geodesic and parallel foliation, and by construction its leaves are the orbits of the action $\Phi$.

Finally, eval ${ }_{x} \circ X: \mathrm{R}^{p} \longrightarrow F_{x}$ is an isometry for any $x \in M$, and this map is just the tangent map at the origin of the map $\Phi_{x}: \mathrm{R}^{p} \longrightarrow M$, so this map is a local isometry from $\mathbf{R}^{p}$ onto the orbit of the point $x$.

\section{Final remarks.}

The last two theorems suggest that on a compact oriented Riemannian manifold the spaces $H_{0}^{p}$ are in general very small, much smaller than the spaces $H^{p}$ of harmonic forms. In fact, the last theorem shows that the flat torus is the only compact oriented surface admitting non-trivial 1 -forms $\omega$ with $\Delta_{0} \omega=0$. But this is not necessarily so in higher dimensions: On a compact connected lie group endowed with an invariant Riemannian metric, all invariant forms are already in $H_{0}$, so in this case $H_{0}^{p}=H^{p}$ in any degree $p$. But $H_{0}^{p} \neq 0$ for $p>1$ does not imply the existence of geodesic and paraliel $p$-dimensional foliations.

\section{References}

1. Goldberg, S.I., "Curvature and Homology," New York-London, 1962.

2. KoBayashi, S. AND K. NOMIZU, "Foundations of Differential Geometry, Vol. 1," New York-London, 1963.

3. YANO, K. AND S, BOCHNER, "Curvature and Betti Numbers," Princeton, 1953 .

Institut de Mathématiques

I'Université de Fribour'g

Chemin du Musée 23

CH-1700 Fribourg, SUISSE

Rebut el 3 de Novembre de 1988 